(c) American Dairy Science Association, 2006.

\title{
Evaluation of the Voluntary Johne's Disease Herd Status Program as a Source of Replacement Cattle
}

\author{
D. A. Kovich, ${ }^{*}$ S. J. Wells, ${ }^{* 1}$ and K. Friendshuht \\ *Department of Veterinary Population Medicine, University of Minnesota, St. Paul 55108 \\ †Minnesota Board of Animal Health, St. Paul 55107
}

\begin{abstract}
The objectives of this study were to evaluate the perceived value that enrolled producers gained from participation in the Voluntary Johne's Disease Herd Status Program (VJDHSP), and to evaluate the risk of infection with Mycobacterium avium ssp. paratuberculosis (MAP) of cattle reared in a presumed Johne's free environment vs. cattle raised in an environment of unknown Johne's status. Producers enrolled in level 3 or 4 of the VJDHSP (98 and 99\% probability, respectively, of being free from MAP infection) were interviewed via telephone. Producers were asked questions pertaining to their participation in the VJDHSP, and asked to identify herds to which they had sold replacement heifers. These cattle were presumed to be uninfected before sale. Fifty-nine cattle (identified as having been purchased into infected herds as heifers) were identified as having been raised in uninfected herds and sold to producers with herds of unknown Johne's disease status. On the purchasing farm, fecal and blood samples were collected from each cow of VJDHSP origin and 3 randomly selected home-reared cows per VJDHSP cow matched by lactation as controls. Samples were tested using commercial ELISA (serum) and bacterial culture (feces). Results indicated that enrolled producers saw value in VJDHSP participation, and that cattle reared in VJDHSP herds were less likely to be infected with MAP than herdmates as measured by serum ELISA MAP antibody and by fecal culture. This study provides evidence of the value of the VJDHSP in providing economic value to participants and supports the promotion of VJDHSP herds as a source of replacement cattle of low infection risk for MAP.
\end{abstract}

Key words: Johne's disease, Mycobacterium avium ssp. paratuberculosis, paratuberculosis, Voluntary Johne's Disease Herd Status Program

Received September 9, 2004.

Accepted May 15, 2006.

${ }^{1}$ Corresponding author: wells023@umn.edu

\section{INTRODUCTION}

Johne's disease (also known as paratuberculosis) is a debilitating diarrheal disease of ruminants caused by Mycobacterium avium ssp. paratuberculosis (MAP). In cattle, clinical signs include progressive diarrhea and weight loss leading to death. The disease is predominantly spread via the fecal-oral route. The disease has a long period of incubation, and clinical signs are rarely seen before $2 \mathrm{yr}$ of age (Whitlock and Buergelt, 1996). Resistance to infection is believed to increase with age. This age-related resistance forms the basis of most control programs, which focus on rearing calves in an environment free from Johne's disease (Goodger et al., 1996).

Johne's disease has a high prevalence in the United States. Nationally, at least $22 \%$ of all dairy herds and $40 \%$ of those having 300 or more cows are believed to be infected. The disease can cost production losses of $\$ 200$ per cow in inventory in heavily infected herds, and the total cost to the US dairy industry exceeds $\$ 200$ million annually (Ott et al., 1999). Furthermore, although the link between MAP and Crohn's disease in humans remains unclear, Johne's disease may be a potential zoonotic concern (Collins, 1997).

Economic costs of Johne's disease and public health concerns have led to the implementation of Johne's disease control programs. The Voluntary Johne's Disease Herd Status Program (VJDHSP) seeks to identify and categorize herds presumed free of Johne's disease. One objective of the program is to provide a source of uninfected replacement heifers for dairy farmers, under the assumption that these cattle in a production setting may be resistant to infection with MAP.

The objectives of this study were to test the hypothesis that enrolled producers perceive an economic advantage to VJDHSP participation, and that cattle introduced to herds before their first parturition are less likely to be detected as infected with MAP on dairy farms than are herdmates raised in infected herds.

\section{MATERIALS AND METHODS}

A list of the 26 Minnesota dairy producers at levels 3 or 4 of the Minnesota VJDHSP was obtained from 
the Minnesota Board of Animal Health (MBAH) in May 2003. Producers at levels 3 and 4 have a 98 and $99 \%$ probability, respectively, of being free from MAP infection. In June and July 2003, up to 5 contact attempts via telephone were made to each producer to collect information regarding sale of replacement cattle and the perceived benefits to participation in the VJDHSP. These questions covered the perceived economic advantages to VJDHSP participation, marketing of replacement cattle to other producers, and motivation for VJDHSP involvement. Sale records of replacement cattle sold by these herds were obtained, and 4 herds were identified as having previously purchased 5 or more heifers from VJDHSP herds and willing to participate in the next phase of the study.

The 4 producers participating in the second phase of the study had purchased cattle just before first calving from level 3 or 4 VJDHSP herds, hereafter referred to as the VJDHSP birth cohort. For each VJDHSP birth cohort cow, 3 home-raised cows of the same lactation number (first, second, or third and greater) were selected randomly as controls, hereafter referred to as the control birth cohort. Control birth cohort selection was accomplished via systematic random sampling matched by lactation number with VJDHSP birth cohort cows. If sufficient control birth cohort cows were not available within a given lactation, cows were selected randomly from the previous (if available) or following lactation. If preselected control birth cohort cows were unavailable at the time of testing, another cow of the same lactation number was randomly selected as replacement. Fecal grab samples were collected from the rectum of each cow using a disposable plastic sleeve. Blood samples were collected from VJDHSP birth cohort and control birth cohort cows by caudal venipuncture. From each sampled cow, BCS ( 1 to 5 scale, $1=$ excessively thin to $5=$ obese) and fecal consistency score (FCS; 1 to 3 scale, $1=$ liquid consistency to $3=$ solid) also were evaluated.

Collected blood and fecal samples were submitted to the Minnesota Veterinary Diagnostic Laboratory for analysis. Blood collected was tested using a commercial serum ELISA test (Idexx, Westbrook, ME) for antibodies to MAP (Collins and Sockett, 1993). Fecal samples were tested by bacterial culture using Herrold's egg yolk medium and a 72-h sedimentation procedure to concentrate the organism.

Results of diagnostic tests were analyzed both for the total population and within lactation number, taking into account the matched case-control study design. The BCS on a scale of 1 to 5 and FCS on a scale of 1 to 3 were summed as an indicator of clinical disease, with any cow scoring 3 or less out of a possible of 8 considered positive for clinical signs of Johne's disease. Relative risks to evaluate associations between cattle being reared in a VJDHSP vs. an environment of unknown infection status and a positive test result for ELISA antibody detection, fecal culture status, and combined BCS and FCS were estimated by the Mantel-Haenszel procedure to account for the matched study design (Mantel and Haenszel, 1959). Relative risks, confidence intervals, and two-sided Fisher's exact $P$-values were calculated using Stata (Statacorp LP, College Station, TX) software. In addition, an association between BCS and FCS and test results was evaluated using contingency tables to calculate relative risk and $95 \%$ confidence intervals.

\section{RESULTS}

Survey results for dairy producers currently enrolled at level 3 or 4 of the MBAH VJDHSP are summarized in Table 1. Results indicated that $90 \%$ of enrolled producers perceived an economic advantage to participation in the VJDHSP, and that 95\% felt that the advantages of the program outweighed associated costs. Reasons for participation in the VJDHSP varied, with the largest percentage of producers (43\%) citing herd health as the motivating factor. Other responses were marketability of surplus cattle (29\%), free testing (19\%), and consumer health (10\%). A majority (71\%) of respondents sold replacement cattle to other producers. Of these, $73 \%$ marketed cattle as being from a VJDHSP herd, 73\% reported that VJDHSP status made replacement cattle easier to sell, and 53\% received a VJDHSP status related premium.

At the time of the survey administration, 15 (71\%) of the respondents were selling replacement cattle. The remaining 6 were not selling cattle because of herd expansion. In total, 461 cattle were identified as having been sold from these herds since 1998. Mean number of cattle sold per producer was 21.1 (median $=18$ ) with a range from 0 to 65 . All 15 producers selling replacement cattle reported that they were sold as springing heifers. Seven of the 15 producers were able to report names of purchasers. Of the 8 that could not name purchasers, 3 sold their cattle internationally (to Italy and China), 3 sold their cattle through a third party, and 2 felt that the information was confidential.

Use of VJDHSP status as a marketing tool was reported by $11(73 \%)$ of the producers selling cattle. They indicated a growing demand for their cattle and that presumed freedom from Johne's disease was important to their customers. This assumption was corroborated by the fact that $73 \%$ of producers selling replacement cattle felt that their VJDHSP status made selling replacement cattle easier. The remaining $27 \%$ reported that demand for replacement cattle was sufficient so 
Table 1. Results of survey of dairy producers at level 3 or 4 of the Minnesota Voluntary Johne's Disease Herd Status Program (VJDHSP)

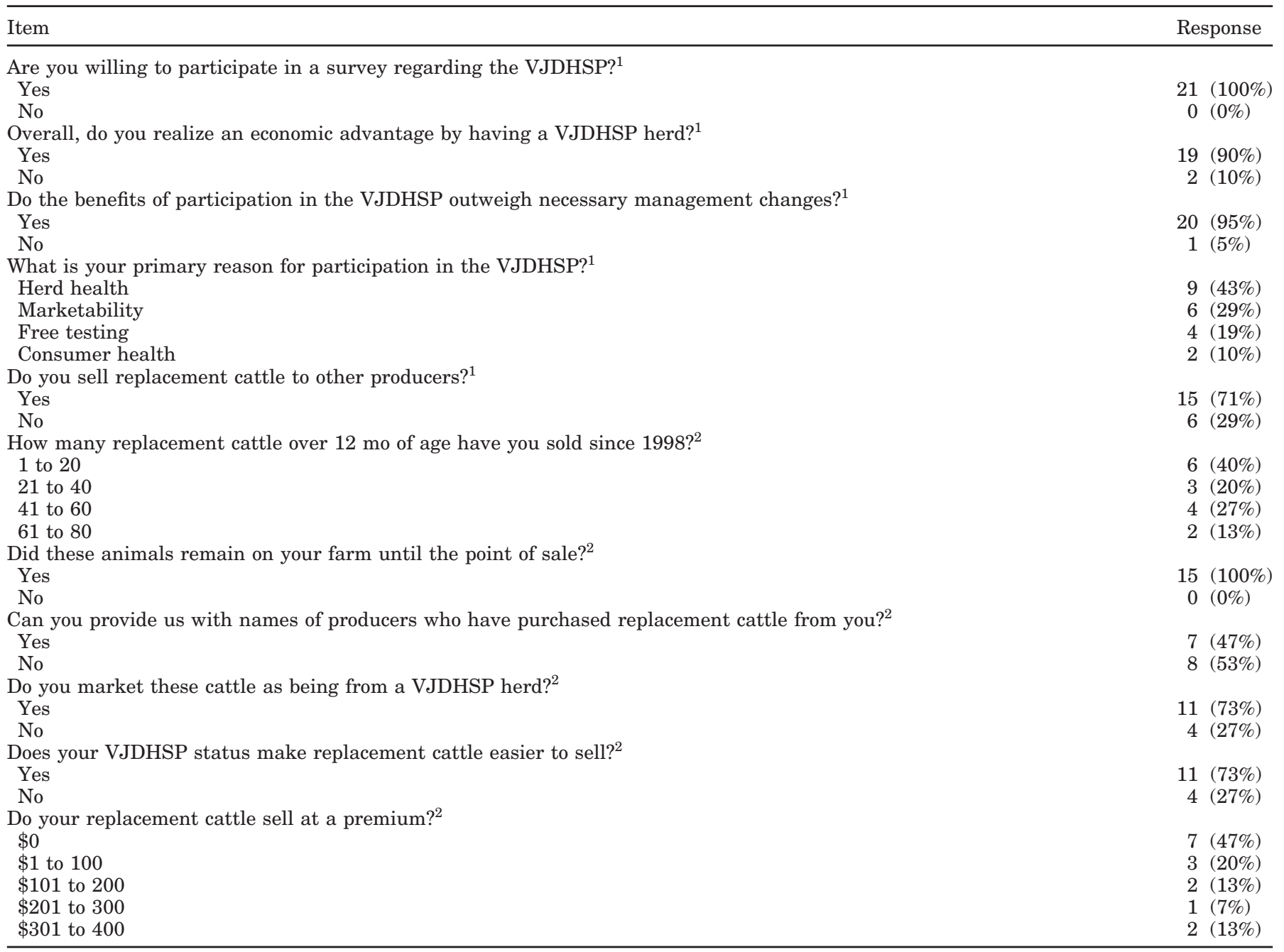

${ }^{1} \mathrm{n}=21$.

${ }^{2} \mathrm{n}=15$ (question was only asked of respondents selling replacement cattle).

that disease status was not a significant factor. Of those selling cattle, $53 \%$ did so at a premium. Mean premium received was $\$ 190.63$ with a range of $\$ 50$ to $\$ 400$.

Test results for the 4 study herds purchasing replacement cattle from dairy producers enrolled at level 3 or 4 of the MBAH VJDHSP are summarized by herd and lactation number for the total sample population in Table 2. These herds had a total of 59 cattle $(5,14,19$, and 21 in each herd) in first through fourth lactations purchased from VJDHSP producers since 1998. Cows raised in infected herds were more likely to test positive to antibody to $M$. paratuberculosis using ELISA than those raised in VJDHSP herds (relative risk $=8.67$; $95 \%$ confidence interval $=1.20$ to $62.5, P=0.004$ ). Of the 59 VJDHSP birth cohort cows, only 1 cow was ELISA positive with a sample to positive ratio of 1.30 . There were 26 ELISA-positive control birth cohort cows with a mean sample to positive ratio of 1.17 (range 0.26 to 2.25). Although trends were observed, associations within lactation number were not statistically significant. Of cows in first lactation, 0 of 21 VJDHSP birth cohort cows tested ELISA positive compared with 9 of 63 control birth cohort cows. For the second lactation, 1 of 22 VJDHSP birth cohort cows tested positive by ELISA vs. 9 of 66 control birth cohort cattle. For the third lactation and greater, 0 of 16 VJDHSP birth cohort cows were ELISA positive compared with 8 of 48 control birth cohort cows.

Cows raised in infected herds also were more likely $(P<0.05)$ to shed detectable $M$. paratuberculosis using bacterial culture of feces than those raised in VJDHSP herds (relative risk $=2.33 ; 95 \%$ confidence interval $=$ 0.96 to 5.68). Of the 59 VJDHSP birth cohort cows, 5 cows were culture positive, all with $<10$ colonies per 
Table 2. Results of diagnostic tests in Voluntary Johne's Disease Herd Status Program (VJDHSP) and control birth cohorts

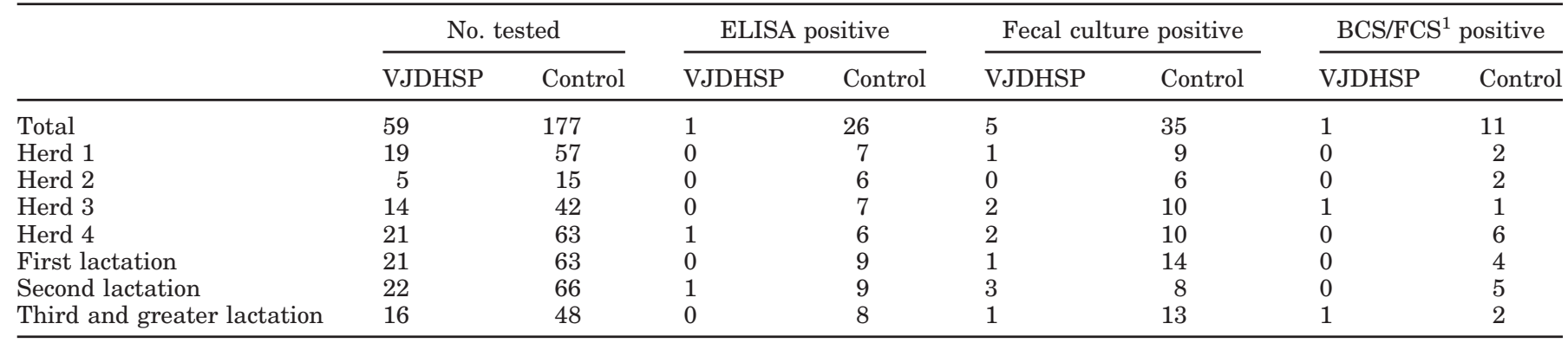

${ }^{1}$ Combined BCS and fecal consistency (FCS) scores.

tube. There were 35 culture positive control birth cohort cows with mean colony scores from $<10$ to $>100$ colonies per tube. Associations between birth cohort and fecal culture results were not significant by lactation number, though the direction of the associations was consistent. For first lactation, 1 of 21 VJDHSP birth cohort cows were fecal culture positive vs. 14 of 63 control birth cohort cows. For second lactation, 3 of 22 VJDHSP birth cohort and 8 of 66 control birth cohort cows were fecal culture-positive. For third lactation or greater, 1 of 16 VJDHSP birth cohort and 13 of 48 control birth cohort cows were fecal positive.

No association was detected between birth cohort and combined BCS and FCS (relative risk $=3.67 ; 95 \%$ confidence interval 0.48 to 27.8). Cattle with a combined BCS and FCS score of 3 or less were more likely to have a positive ELISA result (relative risk $=4.24 ; 95 \%$ confidence interval 1.95 to 9.24 ) and to have a positive fecal culture (relative risk 3.96; 95\% confidence interval 2.23 to 7.02 ).

\section{DISCUSSION}

Results of the survey of dairy producers currently enrolled at level 3 or 4 in the MBAH VJDHSP indicated that there was economic value to participation in the program. Most respondents reported that this benefit was related to increased productivity as a function of better cow health, but for several, the dominant factor was premiums received from surplus replacement cattle sold. Respondents (95\%) felt that the benefits of VJDHSP participation outweighed the management changes (i.e., calving management, manure disposal, and testing regimen) required to achieve and maintain their current status level in the program. Although only 2 producers listed concern over consumer health as their primary reason for participation in the VJDHSP, most other respondents also cited this as a key factor in their decision to participate. The proposed link between Johne's disease in cattle and Crohn's disease was the foundation for this concern. As one advantage of the
VJDHSP is to provide a source of low-infection risk replacement cattle to the dairy industry (Bulaga, 1998), enrolled producers felt poised to take advantage of the demand for these cattle if the link is validated.

The second phase of the study evaluated the assumption that purchasing replacement cattle from a VJDHSP herd reduced the likelihood of the purchased animals developing Johne's disease. This assumption is largely based on previous work demonstrating adult resistance to experimentally induced infection. Early work on adult infection suggested that adult cattle were incapable of developing active infection (Hagan, 1938). Subsequent studies using more sophisticated techniques concluded that MAP was capable of entering the tissue of adult cattle, but not progressing to active infection (Payne and Rankin, 1961) and that adult cattle were more resistant to MAP infection and lesions less pronounced (Larsen et al., 1975). Resistance of adult cattle to field infection with MAP, however, has been little studied. This study provides evidence that adult infection with MAP is possible, particularly in heavily infected herds, even for cattle with no calfhood exposure.

Study results indicated that Johne's disease was prevalent on all 4 farms tested. Cows born and raised in infected herds were more likely to test positive using ELISA than cows born and raised in VJDHSP herds. The 1 VJDHSP birth cohort cow that tested ELISA positive was a second-lactation cow that also tested positive for MAP using fecal culture. Of the 63 control birth cohort cows tested in this same herd, 6 were ELISA positive ( 4 were also fecal culture positive). Association between birth cohort and fecal culture results for the entire sample population was borderline statistically significant. The culture-positive VJDHSP birth cohort cows came from 3 of the 4 herds tested and were in various lactations, with some being in their first lactation. Four of the 5 culture-positive VJDHSP birth cohort cows and 15 of the 35 culture-positive control birth cohort cows were ELISA negative, and these cows all were light fecal shedders. It is possible that in heav- 
ily contaminated environments, cattle can ingest and passively shed MAP before or exclusive of development of infection and development of detectable antibody to MAP. This ability has been demonstrated experimentally (Sweeney et al., 1992).

The FCS and BCS scores were summed as an indicator of clinical disease, as a cow with both a low BCS and a loose fecal consistency would be indicative of possible clinical Johne's disease. Although diarrhea can precede loss of body condition in Johne's disease, disease is generally more apparent when both are present. As expected, cattle that were both thin and had diarrhea were more likely to test positive to both diagnostic tests. Birth cohort did not, however, influence the likelihood of a cow having a low BCS or FCS.

Study results indicated that cattle raised in a VJDHSP herd are less likely to be infected with MAP later in life. Results do not indicate that they are immune to infection. The testing procedure required to reach level 3 or 4 in the VJDHSP makes it sufficiently unlikely that the VJDHSP birth cohort cows were infected with MAP before introduction to the sampled herds (Bulaga, 1998). Our results indicated that farms sampled had a marked prevalence of MAP with an expected high degree of environmental contamination. Some infection with MAP for VJDHSP birth cohort cows after introduction based on our study results seems probable. Results also indicate, however, that in heavily infected herds, purchase of replacement cattle from a VJDHSP herd does offer a lesser infection risk for MAP than home-reared cattle.

\section{CONCLUSIONS}

Dairy producers enrolled at level 3 or 4 in the MBAH VJDHSP perceive an economic advantage in participating in the program, and report that program benefits outweigh necessary management changes. Furthermore, the VJDHSP seems to fulfill one of the stated goals of the program in providing low infection risk replacement cattle to other producers. This information will be of value in both promoting the VJDHSP and ensuring its continuance.

These results indicated that cattle reared in VJDHSP herds, even after residing in infected herds for variable periods, are less likely to develop Johne's disease as measured by detectable antibody to MAP and by detectable fecal shedding. The VJDHSP birth cohort cows also shed less MAP on a per-cow basis than cows born and raised in infected herds $(<10$ colonies/culture tube for all VJDHSP cattle vs. 100 or more colonies/culture tube for control birth cohort cattle). The large number of fecal culture-positive cows that were ELISA negative is suggestive that passive shedding of MAP may occur in highly contaminated environments. Results did not indicate a significant difference in manifestation of clinical disease within birth cohorts as measured by body condition and fecal consistency scores.

The results of this study suggest that cattle raised in VJDHSP herds are less likely to be infected with MAP leading to Johne's disease after introduction to infected herds than herdmates, demonstrating value of the VJDHSP. To measure the protective benefit of being reared in a VJDHSP herd over time, future plans include continued prospective monitoring of study cattle.

\section{REFERENCES}

Bulaga, L. L. 1998. US voluntary Johne's disease herd status program for cattle. Proc. US Anim. Health Assoc. 102:420-433.

Collins, M. T. 1997. Mycobacterium paratuberculosis: A potential food-borne pathogen? J. Dairy Sci. 80:3445-3448.

Collins, M. T., and D. C. Sockett. 1993. Accuracy and economics of the USDA-licensed enzyme-linked immunosorbent assay for bovine paratuberculosis. J. Am. Vet. Med. Assoc. 173:478-480.

Goodger, W. J., M. Collins, K. Nordlund, C. Eisele, J. Pelletier, C. Thomas, and D. Sockett. 1996. Epidemiologic study of on-farm management practices associated with prevalence of Mycobacterium paratuberculosis infections in dairy cattle. J. Am. Vet. Med. Assoc. 208:1877-1881.

Hagan, W. A. 1938. Age as a factor in susceptibility to Johne's disease. Cornell Vet. 28:34-40.

Larsen, A. B., R. S. Merkal, and R. C. Cutlip. 1975. Age of cattle as related to infection with Mycobacterium paratuberculosis. Am. J. Vet. Res. 36:255-257.

Mantel, N., and W. Haenszel. 1959. Statistical aspects of the analysis of data from retrospective studies of disease. J. Natl. Cancer Inst. 22:719-748.

Ott, S. L., S. J. Wells, and B. A. Wagner. 1999. Herd-level economic losses associated with Johne's disease on US dairy operations. Prev. Vet. Med. 40:179-192.

Payne, J. M., and J. D. Rankin. 1961. A comparison of the pathogenesis of experimental Johne's disease in calves and cows. Res. Vet. Sci. 2:175-179.

Sweeney, R. W., R. H. Whitlock, A. N. Hamir, A. E. Rosenberger, and S. A. Herr. 1992. Isolation of Mycobacterium paratuberculosis after oral inoculation in uninfected cattle. Am. J. Vet. Res. 53:1312-1314.

Whitlock, R. H., and C. Buergelt. 1996. Preclinical and clinical manifestations of paratuberculosis (including pathology). Vet. Clin. North Am. Food Anim. Pract. 12:345-356. 